\title{
SEPARATION OF LINEAR AND NONLINEAR MODES IN A HYPERBOLIC SYSTEM DESCRIBING ELECTROPHORESIS
}

\author{
BY
}

\section{HEINRICH FREISTÜHLER}

\section{Institut für Mathematik, Technische Hochschule Aachen, Germany}

For $k \in \mathbb{N}$ and appropriate $\mathscr{D}$ let $\mathscr{P}^{k}(\mathscr{D})$ denote the set of all functions $u=$ $\left(u_{1}, \ldots, u_{k}\right)$ on $\mathscr{D}$ such that each component $u_{i}, 1 \leq i \leq k$, is measurable, positive, and bounded away from 0 and $\infty$, uniformly on $\mathscr{D}$. For any fixed integer $n \geq 2$ and positive coefficients $a_{1}<\cdots<a_{n}$, the equations

$$
\left(u_{i}\right)_{t}+\left(\frac{a_{i} u_{i}}{\sum_{j=1}^{n} u_{j}}\right)_{x}=0, \quad 1 \leq i \leq n,
$$

constitute a hyperbolic system of conservation laws, to be satisfied, weakly, by functions $u \in \mathscr{P}^{n}(\mathbb{R} \times I)$ on some space-time strip $\mathbb{R} \times I \ni(x, t), I \subset \mathbb{R}$ a (nondegenerate) interval. This system, in fact a model for electrophoretic separation of $n$ species of ions (with scaled concentrations $u_{i}$ and relative mobilities $a_{i}$ ), has recently been studied mathematically by Geng [13], Dafermos and Geng [14], and Serre [15]. The goal of the present note is to point out the following observation.

LEMMA. There is a one-to-one correspondence between the weak solutions $u \in$ $\mathscr{P}^{n}(\mathbb{R} \times I)$ of $(1)$ and pairs $(p, q) \in \mathscr{P}^{1}(\mathbb{R}) \times \mathscr{P}^{n-1}(\mathbb{R} \times I)$ with $q$ a weak solution of a certain fixed system of $n-1$ conservation laws associated with (1). More precisely, any function $p \in \mathscr{P}^{1}(\mathbb{R})$ establishes a bijection between the set of all weak solutions $q=\left(q_{1}, \ldots, q_{n-1}\right) \in \mathscr{P}^{n-1}(\mathbb{R} \times I)$ of the system

$$
\left(q_{j}\right)_{t}-\left(\frac{q_{j+1}}{q_{1}}\right)_{y}=0, \quad 1 \leq j \leq n-1, \quad\left(q_{n}: \equiv 1\right)
$$

and those weak solutions $u \in \mathscr{P}^{n}(\mathbb{R} \times I)$ of (1) for which

$$
\left(\prod_{k=1}^{n} a_{k}\right) \sum_{i=1}^{n} \frac{u_{i}(x, t)}{a_{i}}=p(x) \quad \text { a.e. }(x, t) \in \mathbb{R} \times I .
$$

This bijection is given by the transformation

$$
q_{j}\left(\int_{0}^{x} p(\xi) d \xi, t\right)=\frac{m_{j}(x, t)}{p(x)}, \quad \text { a.e. }(x, t) \in \mathbb{R} \times I, \quad 1 \leq j \leq n-1,
$$

Received September 4, 1991.

1991 Mathematics Subject Classification. Primary 35L65, 35A22. 
with

$$
m_{j}=\sum_{i=1}^{n}\left(\sum_{K \in \mathscr{K}_{i j}}\left(\prod_{k \in K} a_{k}\right)\right) u_{i}, \quad 1 \leq j,
$$

where $\mathscr{K}_{i j}:=\{K \subset\{1, \ldots, n\} ; K \not \supset i$ and $K$ has $j-1$ elements $\}$.

This transformation property has, e.g., the following

CONSEQUences. (i) The separation into $p$ and $q$ means an uncoupling of the linear mode and the nonlinear modes of (1); in particular, no contact discontinuities can form newly in solutions of (1). (ii) The solution theory of (1) is equivalent with that of (2); in particular, the results of the uniqueness and regularity theory given in [14] for solutions of (1) with $\sum_{i=1}^{n} \frac{u_{i}}{a_{i}} \equiv$ const hold without this restriction. (iii) (Cf. [14].) The solution theory of (1) is equivalent with that of the system

$$
\left(v_{i}\right)_{t}+\left(\frac{b_{i} v_{i}}{1+\sum_{j=1}^{\nu} v_{j}}\right)_{x}=0, \quad 1 \leq i \leq \nu
$$

of $\nu$-species chromatography (which has been studied in $[7,8,9,10,14]$ ), with $\nu=n-1$. (iv) Two-species electrophoresis ((1) with $n=2$ ) is governed by the scalar conservation law

$$
q_{t}-\left(\frac{1}{q}\right)_{y}=0, \quad q>0
$$

to which the classical Lax-Oleinik theory $([4,5])$ applies.

Proof of the Consequences. (i) The pair $(\hat{p}, q)$ with $\hat{p}\left(\int_{0}^{x} p(\xi) d \xi, t\right):=p(x)$ satisfies (2) and

$$
\hat{p}_{t}=0
$$

The uncoupled trivial equation (8) recovers the linear mode; (4) retains the $n-1$ genuinely nonlinear modes. (For the issues of strict hyperbolicity and genuine nonlinearity/linear degeneracy, see [7, 14, 15].) (ii) Fix $p$ arbitrarily, or, in particular, as any constant. (iii) That the latter is equivalent with studying (6) was pointed out in [14]: Each $v_{i}$ can be chosen as a certain multiple of $u_{i}$, for $1 \leq i \leq n-1$, and the coefficients $b_{1}, \ldots, b_{n-1}$, again positive and pairwise distinct, transform from the coefficients $a_{1}, \ldots, a_{n}$ and the (constant) value of $\sum_{i=1}^{n} \frac{u_{i}}{a_{i}}$. (iv) For $n=2$, $q \in \mathscr{P}^{1}(\mathbb{R})$ and (1) transforms to (7) and (8). The flux function of (7) is strictly concave.

Proof of the Lemma. Let $u \in \mathscr{P}^{n}(\mathbb{R} \times I)$ be a weak solution of (1). Note first (cf. [14]) that the functions $m_{j}$ given by (5) satisfy

$$
\left(m_{j}\right)_{t}-\left(\frac{m_{j+1}}{m_{1}}\right)_{x}=0
$$


this follows as

$$
\begin{aligned}
0 & =\left(\sum_{i=1}^{n}\left(\sum_{K \in \mathscr{K}_{i j}}\left(\prod_{k \in K} a_{k}\right)\right) u_{i}\right)+\left(\frac{\sum_{i=1}^{n} a_{i}\left(\sum_{K \in \mathscr{K}_{i j}}\left(\prod_{k \in \mathscr{K}} a_{k}\right)\right) u_{i}}{\sum_{i=1}^{n} u_{i}}\right)_{x} \\
& =\left(m_{j}\right)_{t}+\left(\frac{1}{m_{1}} \sum_{i=1}^{n}\left(\sum_{K \in \mathscr{K}_{i j}}\left[\sum_{l \notin K} a_{l}-\sum_{l \notin K \cup\{i\}} a_{l}\right]\left(\prod_{k \in K} a_{k}\right) u_{i}\right)\right)_{x} \\
& =\left(m_{j}\right)_{t}+\left(\frac{1}{m_{1}} \sum_{i=1}^{n}\left(\left[\sum_{K \in \mathscr{K}_{0(j+1)}}\left(\prod_{k \in K} a_{k}\right)-\sum_{K \in \mathscr{K}_{i(j+1)}}\left(\prod_{k \in K} a_{k}\right) u_{i}\right)\right)_{x}\right. \\
& =\left(m_{j}\right)_{t}-\left(\frac{m_{j+1}}{m_{1}}\right)_{x} .
\end{aligned}
$$

Next observe that, because of $m_{j} \equiv 0, j>n$, and with $p \equiv m_{n}$-this is (3)-, (9) really means

$$
\begin{aligned}
\left(m_{j}\right)_{t}-\left(\frac{m_{j+1}}{m_{1}}\right)_{x} & =0, \quad 1 \leq j \leq n-1, \\
p_{t} & =0 .
\end{aligned}
$$

Introduce now the "generalized particle coordinate"

$$
y=\int_{0}^{x} p(\xi) d \xi
$$

as a new independent spatial variable, and assign to any function $g$ on $\mathbb{R}$ its corresponding pull-back $\hat{g}$ by

$$
\hat{g}(y)=g(x) \text { with }(12) \text {. }
$$

By the transformation consisting of (12) and (13), system (11), together with the trivial conservation law $1_{t}+0_{x}=0$, become

$$
\begin{aligned}
\left(\frac{\hat{m}_{j}}{\hat{p}}\right)_{t}-\left(\frac{\hat{m}_{j+1}}{\hat{m}_{1}}\right)_{y} & =0, \quad 1 \leq j \leq n-1, \\
\left(\frac{1}{\hat{p}}\right)_{t} & =0 .
\end{aligned}
$$

Setting

$$
q_{j}:=\frac{\hat{m}_{j}}{\hat{p}}=\frac{\hat{m}_{j}}{\hat{m}_{n}}, \quad 1 \leq j \leq n,
$$

we arrive at (2) and (8).

It remains to show that the point transformation which is given by (5) with $1 \leq$ $j \leq n$ is invertible; this, however, follows from the fact that the Jacobian determinant of the flux function of (2), which assumes the value $q_{1}^{-n}$, is different from 0 .

REMARKs. (i) The linear change (5) of dependent variables and the resulting system (9) have appeared in the paper [14] of Dafermos and Geng. (ii) The change (12) of independent variables is analogous to the familiar transition from spatial to material coordinates in fluid mechanics, where $p=p(x, t)>0$ is the density and 
the curves $\left\{(x, t) ; y=\int^{x} p(\xi, t) d \xi=\right.$ const $\}$ are particle paths, and goes, in this sense, back to Euler and Lagrange. (Besides formulating the fundamental equations of fluid flow in spatial coordinates (the "Eulerian" description), Euler was also the first, in [1], to adopt the material ("Lagrangian") coordinates point of view; in contrast to Euler's kinematic approach, Lagrange's re-derivation, in [2], of the equations in these coordinates was based on the idea of formally transforming from spatial to material coordinates by means of the chain rule. Cf. Part XVII of [3].) In the modern framework of hyperbolic systems of conservation laws ([4]), this kind of change of coordinates appears as a transformation of the differential equations, which is associated, as a possibility, with any a priori semi-bounded conserved quantity (" $p$ "), and its technical validity for weak solutions is expressed in Theorem 2 of [11], or Proposition 5.1 of [12]. Was Gel' fand, in [6], the first to formulate the possibility of such transformations for general systems of conservation laws?

Acknowledgment. I thank Professors C. Dafermos and D. Serre for giving me papers [14] and [15] as preprints. The fact that [6] contains a paragraph on generalized particle coordinates has been pointed out to me by Professor Li T.-T.

\section{REFERENCES}

[1] L. Euler, Lettre de M. Euler à M. de la Grange, Recherches sur la propagation des ébranlements dans un milieu élastique, Misc. Taur. $2^{2}$ (1760-1761) 1-10, 1762

[2] J.-L. Lagrange, Application de la méthode exposée dans le mémoire précédent à la solution de différents problèmes de dynamique, Misc. Taur. $2^{2}$ (1760-1761), 196-298, 1762

[3] C. A. Truesdell, Rational fluid mechanics, 1687-1765 (= Editor's introduction to) Leonhardi Euleri Commentationes ad Theoriam Fluidorum Pertinentes, Leonhardi Euleri Opera Omnia II, 12, Orell Füssl, Zurich, 1954

[4] P. D. Lax, Hyperbolic Systems of Conservation Laws II, Commun. Pure Appl. Math. 10, 537-566 (1957)

[5] O. A. Oleinik, Discontinuous solutions of nonlinear differential equations, Uspekhi Mat. Nauk 12, 3-73 (1957); English transl. in Amer. Math. Soc. Transl. Ser. 2 26, 95-172 (1963)

[6] I. M. Gel' fand, Some problems in the theory of quasilinear equations, Uspekhi Mat. Nauk 14, 87-158 (1959); English transl. in Amer. Math. Soc. Transl. Ser. 2 29, 295-381 (1963)

[7] H. Rhee, R. Aris, and N. R. Amundson, On the theory of multicomponent chromatography, Philos. Trans. Roy. Soc. London A 267, 419-455 (1970)

[8] B. Temple, Systems of conservation laws with invariant submanifolds, Trans. Amer. Math. Soc. 280 (1983)

[9] R. J. Leveque and B. Temple, Stability of Godunov's scheme for a class of $2 \times 2$ systems of conservation laws, Trans. Amer. Math. Soc. 288, 115-123 (1985)

[10] D. Serre, Solutions à variations bornées pour certains systèmes hyperboliques de lois de conservation, J. Differential Equations 68, 137-169 (1987)

[11] D. Wagner, Equivalence of the Euler and Lagrangian equations of gas dynamics for weak solutions, J. Differential Equations 68, 118-136 (1987)

[12] D. Serre, Les ondes planes en électromagnétisme non linéaire, Physica D 31, 227-251 (1988)

[13] X. Geng, The conservation laws in isotachophoresis model, Thesis, University of Arizona, 1990

[14] C. Dafermos and X. Geng, Generalized characteristics, uniqueness, and regularity of solutions in a hyperbolic system of conservation laws, Ann. Inst. H. Poincaré Anal. Non Linéaire 8, 231-269 (1991)

[15] D. Serre, Oscillations non linéaires des systèmes hyperboliques: Méthodes et résultats qualitatifs, Ann. Inst. H. Poincaré Anal. Non Linéaire 8, 351-417 (1991) 